\title{
Instrumentación para sistemas automatizados de medición dinámica de hidrocarburos
}

\author{
Instrumentation for Automated Oil and \\ Gas Dynamic Measurement Systems
}

\author{
Andrés Escobar-Díaz ${ }^{1}$ Luis Eduardo Marín-Oviedo², \\ Harold Vacca-González ${ }^{3} \square$
}

${ }_{1}^{1}$ Universidad Distrital Francisco José de Caldas, Bogotá, Colombia
${ }^{2}$ Universidad Distrital Francisco José de Caldas, Bogotá, Colombia
${ }^{3}$ Universidad Distrital Francisco José de Caldas, Bogotá, Colombia

Transversal 70B n. ${ }^{\circ} 73 \mathrm{~A}-35$ Sur, Facultad Tecnológica, Universidad Distrital Francisco José de Caldas, Bogotá, Colombia. Correo electrónico: aescobar@correo.udistrital.edu.co

Recibido: enero 25 del $2018 \quad$ Aprobado: mayo 10 del $2018 \quad$ Disponible en línea: septiembre 1 del 2018

Cómo citar este artículo: A. Escobar-Díaz, L. E. Marín-Oviedo, H. Vacca-González, "Instrumentación para sistemas automatizados de medición dinámica de hidrocarburos", Revista Ingeniería Solidaria, vol. 14, no. 26, 2018. doi: https://doi. org/10.16925/in.v14i24.2306

\section{Resumen}

Introducción: el artículo establece una revisión conceptual sobre la instrumentación utilizada en sistemas automáticos de medición de hidrocarburos, realizada por los grupos de investigación ORCA y SciBas de la Universidad Distrital Francisco José de Caldas en el año 2017.

Problema: ausencia en la literatura sobre el concepto transferencia de custodia (Tc) y su impacto en la cantidad y la calidad de un hidrocarburo en un sistema de distribución.

Objetivo: conceptualizar acerca de la instrumentación en mediciones de alto volumen, en particular las de tipo dinámico.

Metodología: selección, priorización e interpretación de fuentes disponibles en el sector industrial -amparadas por el Instituto Americano del Petróleo (API) y la Asociación Americana de Gas (AGA)- para definir la instrumentación de un sistema confiable y preciso de medición del hidrocarburo.

Resultados: conceptualización sobre unidades del API y la AGA con respecto a equipos LACT (Lease Automatic Custody Transfer), sobre criterios de determinación de la instrumentación para sistemas de Tc, y sobre equipos de medición en una distribución de instrumentación.

Conclusión: obtención de una línea de base conceptual sobre problemáticas de Tc y su instrumentación en un sistema automático de medición dinámica de hidrocarburos.

Originalidad: dentro de la literatura académica es escasa la referencia a revisiones sobre equipos e instrumentación que se utilizan en los sistemas automáticos para Tc de hidrocarburos.

Limitaciones: como los caudalímetros son los instrumentos más importantes del patín de medición con respecto a los revisados, se han de incluir -en perspectiva- otros de efecto pero que usan algoritmos adaptativos de medida -como las redes neuronales (RN), por ejemplo-.

Palabras clave: automatización, gas natural, instrumentación, transferencia de custodia. 


\title{
Instrumentation for Automated Oil and Gas Dynamic Measurement Systems
}

\author{
Abstract \\ Introduction: The article establishes a conceptual review on the instrumentation used in automatic oil and gas \\ measurement systems, carried out by the ORCA and SciBas research groups at the Universidad Distrital Francisco \\ José de Caldas in 2017. \\ Problem: Absence in the literature of the concept of custody transfer (CT) and its impact on the quantity and quality \\ of gas and oil in a distribution system. \\ Aim: To conceptualize about instrumentation in high volume measurements, particularly those of dynamic type. \\ Methods: Selection, prioritization and interpretation of sources available in the industrial sector-covered by the \\ American Petroleum Institute (API) and the American Gas Association (AGA) - to define the instrumentation of a \\ reliable and accurate gas and oil measurement system. \\ Results: Conceptualization of API and AGA units with respect to LACT (Lease Automatic Custody Transfer) equipment, \\ of criteria for determining the instrumentation for ст systems, and of measurement equipment in instrumentation \\ distribution. \\ Conclusion: Obtaining of a conceptual baseline on cт problems and their instrumentation in an automatic gas and \\ oil dynamic measurement system. \\ Originality: The academic literature contains little reference to reviews on equipment and instrumentation used in \\ automatic systems for oil and gas ст. \\ Limitations: Since flowmeters are the most important instruments of the metering skid with respect to those \\ reviewed, other flowmeters that use adaptive measurement algorithms such as neural networks (NN) should be \\ included -in perspective-.
}

Keywords: automation, natural gas, instrumentation, custody transfer

\section{Instrumentação para Sistemas Automatizados de Medição Dinâmica de Hidrocarbonetos}

\section{Resumo}

Introdução: 0 artigo estabelece uma revisão conceitual sobre a instrumentação utilizada em sistemas automáticos de medição de hidrocarbonetos, realizada pelos grupos de pesquisa oRCA e SciBas da Universidad Distrital Francisco José de Caldas em 2017.

Problema: a falta de literatura sobre o conceito de transferência de custódia (тc) e seu impacto na quantidade e qualidade de um hidrocarboneto em um sistema de distribuição.

Objetivo: conceituar a instrumentação em medições de alto volume, particularmente aquelas do tipo dinâmico.

Metodologia: seleção, priorização e interpretação de fontes disponíveis no setor industrial -apoiadas pelo American Petroleum Institute (API) e pela American Gas Association (AGA)- para definir a instrumentação de um sistema confiável e preciso de medição de hidrocarbonetos.

Resultados: conceituação das unidades do API e da AGA em relação ao equipamento LACT (Lease Automatic Custody Transfer), de critérios que determinam a instrumentação para sistemas de tc e de equipamentos de medição em uma distribuição de instrumentação.

Conclusão: obtenção de uma linha de base conceitual sobre problemas de tc e sua instrumentação em um sistema automático de medição dinâmica de hidrocarbonetos.

Originalidade: dentro da literatura acadêmica, há pouca referência a revisões de equipamentos e instrumentação utilizados nos sistemas automáticos de tc de hidrocarbonetos.

Limitações: dado que os medidores de vazão são os instrumentos mais importantes do skid de medição em relação aos revisados, outros que usam algoritmos de medição adaptativa precisam ser incluídos, como redes neurais (RN), para dar um exemplo.

Palavras-chave: automação, gás natural, instrumentação, transferência de custódia. 


\section{Introducción}

El consumo mundial de energía se incrementó en 2,5\% durante el 2011: a diario se gastaron 88 millones de barriles de petróleo y nueve billones de metros cúbicos de gas natural. La producción de crudo en Colombia, para ese mismo año, fue de 930.000 barriles, mientras que la de gas natural estuvo cercana a los veintinueve millones de metros cúbicos [1], [2]. En consecuencia, en la venta de hidrocarburos debe requerirse una medición confiable y precisa: un leve error, sistemático o aleatorio, representa graves consecuencias económicas que pueden perjudicar, en las transacciones, tanto al vendedor como al comprador. En este contexto, según Dupuis y Hwang [3], los sistemas de medición dinámica son utilizados en la venta de combustibles para medir automáticamente, con exactitud - es decir: a través de una sola medida-, la cantidad entregada. Los lineamientos para la construcción de estos sistemas son establecidos por organizaciones internacionales como el Instituto Americano del Petróleo (API, por sus siglas en inglés).

Dado lo anterior, y ante la escasa literatura al respecto, en este documento se realiza un análisis de los equipos y la instrumentación que se utilizan en los sistemas automáticos para transferencia de custodia de hidrocarburos, revisando e ilustrando los conceptos involucrados. En primer lugar, se define el concepto transferencia de custodia (TC); luego, se caracterizan los sistemas de medición dinámica, distinguiendo las unidades LACT (Lease Automatic Custody Transfer) involucradas para hidrocarburos líquidos y las estaciones de medición de gas; después, se describe la Instrumentación para Sistemas de Transferencia de Custodia y su utilidad de acuerdo con las características generales de los hidrocarburos; luego, se describen los equipos para realizar una medición de calidad y se aplica un ejemplo de distribución de instrumentación; finalmente, se establecen las conclusiones en perspectiva.

\section{Materiales y métodos}

En la industria de hidrocarburos, la TC es aquel proceso por el cual el actual dueño de un combustible cede totalmente la posesión, el control y la responsabilidad de su manejo a un nuevo propietario.
Este cambio de dueño se realiza en un punto del sistema de transporte de hidrocarburos, ya sea intermedio o al final de un oleoducto o gasoducto, según el tipo de combustible. Las mediciones de calidad y volumen obtenidas en la TC son utilizadas con propósitos comerciales y fiscales para conseguir la respectiva facturación de venta, así como el cálculo de impuestos o regalías. Debido al impacto económico que tiene la medición en la TC, es necesario que las mediciones sean de alta precisión minimizando el error, según lo acordado en los contratos [3], [4].

Ante la sensibilidad del tema y la escasa literatura, en consecuencia, la metodología seguida se enmarca en una modalidad de investigación documental — de acuerdo con Londoño et al. [5]—, a partir de la cual se obtiene una revisión conceptual contextualizada de la instrumentación usada en sistemas automatizados de medición dinámica de hidrocarburos. Inicialmente, se focaliza el material documental esencial emitido por el Instituto Americano del Petróleo (API, por sus siglas en inglés) y la Asociación Americana de Gas (AGA, por sus siglas en inglés); luego, se establece una taxonomía en tres categorías: sistemas de medición dinámica determinados por el MPMs (Manual of Petroleum Measurements Standards); unidades LACT; y estaciones de medición de gas. Después, se realiza la etapa de interpretación (hermenéutica) de las fuentes sobre la Instrumentación para Sistemas de Transferencia de Custodia. La figura 1 muestra el modelo metodológico seguido, validado por los grupos de investigación Orca y SciBas.

\section{Sistemas de medición dinámica}

A lo largo de la historia de la industria de hidrocarburos, organizaciones como el API y la AGA han recopilado, documentado y estandarizado las características y los conjuntos de procedimientos que especifican los lineamientos para la óptima instalación y operación de equipos que funcionan en la industria, repasando la cadena de valor y todas las actividades previstas: exploración, producción, refinamiento, transporte y distribución. También, han definido las normas para los componentes de tuberías, válvulas, tanques e instrumentación, incluyendo políticas de seguridad eléctrica, riesgos de incendio, comunicaciones y entrenamientos orientados a la operación y el mantenimiento [6]. 


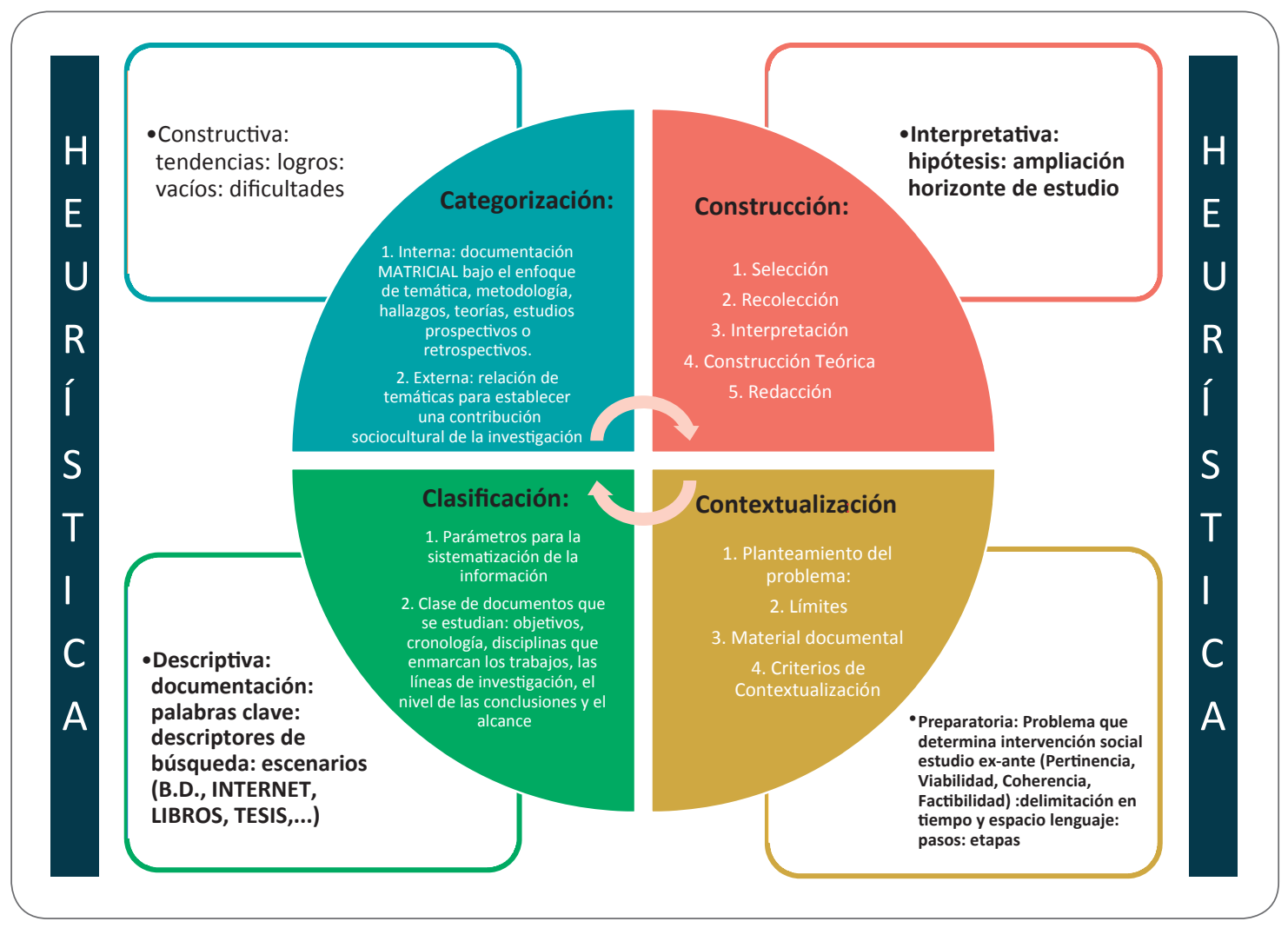

Figura 1. Esquema que describe la metodología de investigación documental seguida para instrumentación de sistemas automatizados en medición dinámica de hidrocarburos Fuente: elaboración propia

Ahora, en cuanto a las normas específicas para sistemas de medición, se deben tener en cuenta los capítulos mencionados en la tabla 1 -que corresponden al MPMS-.

En este contexto, es plausible adoptar un sistema de medición dinámica que no tenga elementos de almacenamiento como tanques o depósitos, de modo que el fluido sea medido mientras fluye continuamente. Tal medición determina la calidad y la cantidad del combustible de manera eficaz cuando funciona las 24 horas del día de forma automática e ininterrumpida [7].

Aunque en Colombia la norma técnica 6167 -la cual establece los requisitos, las directrices y los procedimientos técnicos para realizar la medición de TC de gas natural- apareció en el 2016, la presente investigación se centra en los capítulos exhibidos en las tablas 1,2 y 3.
Tabla 1. Capítulos del MPMs usados en la medición de transferencia de custodia

\begin{tabular}{cc}
\hline MPMS & Título \\
\hline Capítulo 4 & Proving systems \\
Capítulo 5 & Metering \\
Capítulo 6 & Metering assemblies \\
Capítulo 18 & Custody transfer \\
\hline
\end{tabular}

Fuente: elaboración propia

\subsection{Unidades LACT (Lease Automatic Custody Transfer)}

Las unidades LACT realizan automáticamente la transferencia de custodia de hidrocarburos líquidos determinando el volumen neto y la calidad 
del fluido. Estos sistemas se componen de equipos de instrumentación, válvulas y tuberías, montados y ensamblados sobre una estructura metálica denominada "patín". Para diseñar estos sistemas de medición, el API ha definido las normas: Specification for Lease Automatic Custody Transfer (LACT) Equipment [8] y Manual of Petroleum Measurement Standards: Metering Assemblies Section 1 Lease Automatic Custody Transfer (LACT) Systems [9]. El funcionamiento de una unidad LACT inicia cuando el hidrocarburo es bombeado y el fluido es acondicionando eliminando el aire, el agua e impurezas presentes. El caudal, la presión, la temperatura y la densidad del fluido son medidos para calcular y registrar el volumen en condiciones estándar. Finalmente, se ajusta la presión de salida para dispensar el fluido [9].

\subsection{Estaciones de medición de gas}

Estos sistemas de medición son diseñados para determinar la calidad y cantidad de gas natural dispensado en una TC. La medición de gas natural es más compleja que la de hidrocarburos líquidos, porque el gas es un fluido compresible que cambia su volumen en función de su composición, presión y temperatura. Para la construcción de una estación de medición de gas, la AGA establece normas específicas (ver tabla 2) para la medición de calidad y de volumen. La instrumentación del sistema de medición también requiere las normas del API [10], [11] para cumplir los requerimientos de tuberías, instalación de instrumentación, sistema eléctrico y de protección contra fuego.

Tabla 2. Normas de la AGA para medición de gas natural

\begin{tabular}{cl}
\hline \multicolumn{1}{c}{ Norma } & \multicolumn{1}{c}{ Título } \\
\hline AGA Report No. 4 & $\begin{array}{l}\text { Natural Gas Contract Measure- } \\
\text { ment and Quality Clauses }\end{array}$ \\
AGA Report No. 5 & $\begin{array}{l}\text { Natural Gas Energy Measurement } \\
\text { Compressibility Factor of Natural } \\
\text { Gas and Related Hydrocarbon } \\
\text { Gases Report No. } 8\end{array}$ \\
AGA Report No.9 & $\begin{array}{l}\text { Measurement of Gas by Mul- } \\
\text { tipath Ultrasonic Meters } \\
\text { Speed of Sound in Natural Gas } \\
\text { and Other Related Hydrocarbon } \\
\text { Gases }\end{array}$ \\
\hline
\end{tabular}

Fuente: elaboración propia

\section{Instrumentación para sistemas de transferencia de custodia}

En todo proceso industrial, las características de los materiales que requieren ser medidos tienen propiedades que los diferencian entre sí. Del mismo modo, existen diferentes equipos y principios de medición que deben ser empleados para determinar eficientemente estos atributos. Las normas para la instalación de instrumentación se encuentran dadas por el API en la publicación Recommended Practice 551 - Process Measurement Instrumentation [11]; esta a su vez incluye otras consideraciones en cuanto a la instalación eléctrica y las adecuaciones de tuberías.

\subsection{Características generales de los hidrocarburos}

Conviene ahora determinar - para petróleo crudo y gas natural- las características y las unidades de medida en la medición dinámica.

\subsubsection{Características del petróleo crudo}

El petróleo es un combustible fósil extraído del subsuelo. Mediante procesos de fraccionamiento, combinación y destilación, entre otros, se obtiene gran variedad de combustibles y derivados usados en la industria petroquímica. Las características más importantes del petróleo crudo para su medición en unidades LACT son: 1) densidad, 2) viscosidad, 3) contenido de agua y 4) contenido de azufre, según Rey [12].

En cuanto a la gravedad específica (GE) - relación entre el peso del combustible respecto al peso del agua a una temperatura estándar de referencia-, esta se basa en los grados API (notados ${ }^{\circ} \mathrm{API}$ ) que determina una escala de densidad del petróleo propia del Instituto Americano del Petróleo. La ecuación 1 establece en general tal relación:

$$
G E=\frac{141,5}{{ }^{\circ} \mathrm{API}+131,5}
$$

Como se aprecia, cuanto más alto es el índice menor es la densidad del crudo. La mayoría de los crudos tienen una entre 27 y $40^{\circ} \mathrm{API}$. Los inferiores a $27^{\circ}$ API se consideran crudos pesados y los superiores a $40^{\circ}$ API se consideran crudos livianos [13]. 


\subsubsection{Características del gas natural}

El gas natural es una mezcla de hidrocarburos y otras sustancias consideradas impurezas. Se compone en un $95 \%$ de metano $\left(\mathrm{CH}_{4}\right)$ y otros alcanos de mayor peso molecular. Las sustancias no deseadas son el vapor de agua $\left(\mathrm{H}_{2} \mathrm{O}\right)$ y el sulfuro de hidrógeno $\left(\mathrm{H}_{2} \mathrm{~S}\right)$, debido a que disminuyen el poder calorífico y generan daños en las tuberías. El poder energético del gas natural está alrededor de los $1.000 \mathrm{BTU} / \mathrm{ft}^{3}$ (British Thermal Unit/feet ${ }^{3}$ ). En Colombia, en Ecopetrol [14], las especificaciones de calidad del gas natural son definidas por el RUT (Reglamento Único de Transporte de Gas Natural) de la CREg (Comisión de Regulación de Energía y Gas). La tabla 3 indica algunas de las características más importantes del gas natural.

Tabla 3. Características importantes del gas natural

\begin{tabular}{lc}
\hline \multicolumn{1}{c}{ Properties } & Value \\
\hline Carbon content, weight $\%$ & 73.3 \\
Hydrogen content, weight \% & 23.9 \\
Oxygen content, weight \% & 0.4 \\
Relative molar mass & $17-20$ \\
Relative density, $15^{\circ} \mathrm{C}$ & $0.72-0.81$ \\
Boiling point, ${ }^{\circ} \mathrm{C}$ & -162 \\
Auto-ignition temperature, ${ }^{\circ} \mathrm{C}$ & $540-560$ \\
Stoichiometric air/fuel ratio, weight & 17.2 \\
Lower heating/calorific value, $\mathrm{MJ} / \mathrm{kg}$ & $38-50$ \\
Methane concentration, volume $\%$ & $80-99$ \\
Ethane concentration, volume $\%$ & $2.7-4.6$ \\
Nitrogen concentration, volume $\%$ & $0.1-15$ \\
Carbon dioxide concentration, volume $\%$ & $1-5$ \\
Sulfur concentration, weight $\%$ ppm & $<5$ \\
\hline
\end{tabular}

Fuente: Mokhatab et al. [15]

\subsection{Equipos para medición de calidad}

Los equipos para medición de calidad en hidrocarburos determinan características que impactan la capacidad energética. Lo deseable en estas mediciones es que los valores de impurezas tiendan a cero; sin embargo, el producto debe cumplir por lo menos con la conformidad de calidad acordada por las partes en el contrato de TC. Para el petróleo crudo, se debe medir la cantidad de sedimentos y de agua presentes. En el gas natural, se debe medir el contenido de sulfuro de hidrógeno y el de vapor de agua. Los equipos de medición de calidad son considerados de "instrumentación analítica", ya que determinan sustancias específicas con procedimientos de alta tecnología.

\subsubsection{Monitor BSঊW}

El monitor de Bs\&W (Basic Sediment \& Water) es usado en las unidades LACT. Este equipo determina la cantidad de sedimentos y de agua presentes en el petróleo crudo. El principio de funcionamiento de este equipo se basa en la medición de la constante dieléctrica (capacitancia) del caudal. El porcentaje de BS\&W no puede superar el 1\%, según la API [8].

\subsubsection{Higrómetro}

Este equipo mide la cantidad de vapor de agua presente en los hidrocarburos. La presencia de agua en el crudo genera una medición incorrecta de volumen y genera daños en los caudalímetros. En el gas natural, el vapor de agua disminuye el poder calorífico y deteriora las tuberías provocando fugas. Existen diferentes dispositivos de variadas tecnologías para la medición de agua: sensores de impedancia, "chilled mirrors", microbalanzas de cuarzo, interferómetros Fabry-Pérot y espectroscopios con diodo túnel láser (TDLAS), de acuerdo con McKeogh y Soleyn [16].

\subsubsection{Cromatógrafo}

Estos son usados en las estaciones de medición de gas para determinar la concentración de sustancias del gas natural mediante el calentamiento progresivo de la muestra. Los diferentes puntos de ebullición de las sustancias permiten su separación y la cantidad presente es directamente proporcional al tiempo de retención de la sustancia. Los resultados del análisis se representan en un cromatograma, cuya geometría determina la cantidad presente de las sustancias, según Hale [17].

\subsection{Equipos para medición de volumen}

La medición de volumen tiene lugar una vez se ha verificado que el hidrocarburo cumple los criterios de calidad. El volumen se puede obtener 
directamente o de modo inferencial, dependiendo del tipo de caudalímetro usado. Para determinar acertadamente el caudal, de acuerdo con Rey [12], se debe acondicionar el flujo y realizar mediciones complementarias de presión y temperatura para obtener las condiciones estándar establecidas por AGA Y API.

\subsubsection{Eliminador de gas/aire}

Para los hidrocarburos líquidos, los fluidos en fase gaseosa generan mediciones erróneas de caudal. Para remediar esto, se debe eliminar el gas y el aire presentes por medio de una serie de filtros instalados en el acondicionador de flujo. La evacuación se realiza por medio de una válvula de retención (check valve) para evitar que el fluido se devuelva al sistema de medición, según Rey [12].

\subsubsection{Acondicionador de flujo}

Este equipo, denominado "Strainer", es un componente mecánico que tiene funciones diferentes en las unidades LACT y en las estaciones de medición de gas. Para la medición de hidrocarburos líquidos, el Strainer consiste en un filtro que bloquea el paso de sólidos (ver figura 2). Un medidor de presión diferencial deber ser instalado sobre el filtro para determinar si este debe ser limpiado o reemplazado. Para la medición de gas, el acondicionador de flujo ajusta el perfil de flujo del fluido haciéndolo que se comporte de modo laminar, tal como lo registran Francisco [18] y Mokhatab [19].

\subsubsection{Medidores de caudal volumétrico}

La medición de flujo o caudal se puede realizar obteniendo el volumen del fluido de forma directa o inferencial. La medición directa se obtiene por el conteo de pulsos cada vez que el fluido entra y sale de la cámara del caudalímetro. La medición inferencial se realiza midiendo el efecto de otras magnitudes que están relacionadas directamente con el caudal, como las caídas de presión y de velocidad, según Creus [20].

\subsubsection{Caudalímetro de \\ desplazamiento positivo (PD)}

Los caudalímetros PD son utilizados en las unidades LACT. Son dispositivos de tecnología tradicional, con más de cincuenta años de uso. Este tipo de medidor mecánico unidireccional es muy efectivo y de bajo costo en comparación con los caudalímetros modernos como los ultrasónicos (ver figura 3). Miden crudos altamente viscosos a temperaturas entre -32 y $52^{\circ} \mathrm{C}$. El flujo que pueden medir está entre sesenta y 13.000 barriles por hora según el tamaño (de 2" a 16"). La linealidad es de $\pm 0,25 \%$ y la repetibilidad es de $\pm 0,02 \%$. Este medidor volumétrico no es afectado por la velocidad o la viscosidad del producto y no necesita acondicionamiento de flujo, pero requiere de filtros y demanda alto mantenimiento. La señal de salida es una serie de pulsos que corresponden a cada revolución del medidor y requieren de un totalizador que calcula el volumen medido en función de los pulsos, de acuerdo con Rudroff [21] y Yoder [22].

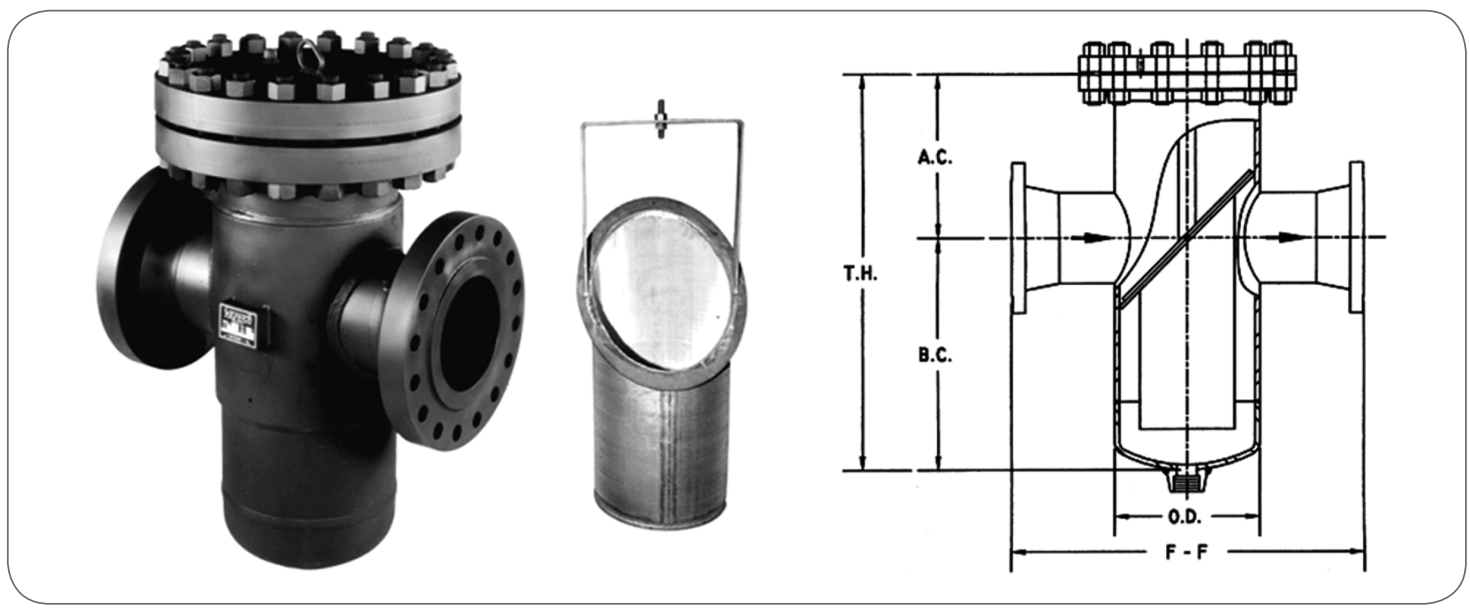

Figura 2. Aspecto físico de un acondicionador (Strainer) tipo canasta y sus cotas Fuente: Mokhatab [19] 


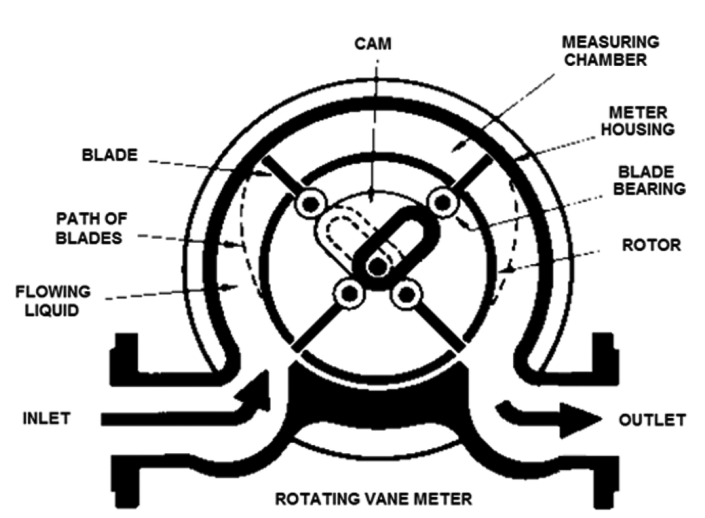

Figura 3. Medidor de caudal por desplazamiento. Cada rotación desplaza un volumen fijo de líquido Fuente: Yoder [22]

\subsubsection{Caudalímetro ultrasónico (UFM)}

Los caudalímetros ultrasónicos son dispositivos con una de las tecnologías más modernas y eficientes disponibles actualmente en el mercado [23]. Son muy usados para la medición de gas debido a que no son intrusivos, reduciendo al máximo las caídas de presión. Miden flujo volumétrico de modo inferencial a partir de la velocidad media del flujo. El uso de este tipo de caudalímetro ha crecido sustancialmente en los últimos años, sobre todo en aplicaciones de transferencia de custodia, poniéndose por delante de las tecnologías tradicionales para medición de gas natural como placa de orificio, desplazamiento positivo y caudalímetro de turbina [24], [25], [26].

A pesar de que su precio de compra inicial es uno de los más elevados, los reducidos costos de operación y mantenimiento representan un alto beneficio económico a mediano y largo plazo. Los caudalímetros ultrasónicos son versátiles en un amplio campo de aplicaciones con diferentes tipos de fluidos y condiciones de presión y temperatura, pero se destacan porque han sido desarrollados especialmente para mediciones de transferencia de custodia, según Spitzer [26] y Dupuis y Hwang [3]. Así mismo, los caudalímetros ultrasónicos han sido estudiados en mayor detalle haciendo que su incertidumbre de medida sea una de las más reducidas, de acuerdo con Helminski [27]; operan comúnmente bajo dos principios de funcionamiento: efecto Doppler (reflexión) y tiempo de tránsito.
La selección del caudalímetro ultrasónico depende del fluido de la aplicación. La medición de gas natural, por ejemplo, se realiza con caudalímetros ultrasónicos que funcionan bajo el principio de tiempo de tránsito [27], [28]. El cuerpo de este tipo de caudalímetro es cilíndrico, con conexión bridada del mismo diámetro de la tubería del proceso, como lo registran Lunde et al. [29].

Los caudalímetros ultrasónicos industriales que operan bajo el principio de tiempo de tránsito miden electrónicamente la diferencia de tiempo que le toma viajar a un pulso acústico de alta frecuencia (beam) en sentidos opuestos (aguas arriba y aguas abajo), a través del canal acústico (path) formado por la trayectoria diagonal entre dos transductores ubicados en las paredes de la tubería (el canal acústico intersecta el eje axial de la tubería con un ángulo específico) [27], [29], [30]. El pulso de sonido viaja más rápido en el sentido del flujo (aguas abajo) y más lento en el sentido opuesto del flujo (aguas arriba). Si no hay flujo, el tiempo de tránsito es igual en ambos sentidos. Asumiendo que el tiempo de tránsito del pulso es proporcional a la velocidad media del flujo, al multiplicarla por la sección transversal de la tubería permite establecer la tasa de caudal volumétrico [27], [28], [29], [30].

Los pulsos ultrasónicos son transmitidos y recibidos por un par de transductores piezoeléctricos que se encuentran instalados en cavidades de las paredes opuestas de la tubería [28]. La velocidad $v$ del flujo se calcula en función del tiempo de tránsito $t$. El método planteado en las ecuaciones 2, 3 y 4 no dependen de la frecuencia del pulso ultrasónico o de las propiedades termofísicas del fluido. La longitud del canal acústico $L$ entre los transductores y el ángulo $\varphi$ respecto al eje axial de la tubería es definida por el fabricante del caudalímetro, como se aprecia en la figura 4 [30].

Tiempo de Tránsito $(A B)$

$$
t_{A B}=\frac{L}{c+v \cdot \cos \varphi}
$$

Tiempo de Tránsito $(B A)$

$$
t_{B A}=\frac{L}{c-v \cdot \cos \varphi}
$$

Velocidad media del fluido

$$
v=\frac{L}{2 \cos \varphi} \cdot\left(\frac{1}{t_{A B}}-\frac{1}{t_{B A}}\right)(9)(4)
$$


Este medidor se considera de tipo inferencial, y el principio de funcionamiento se basa en medir el tiempo de tránsito de pulsos de ultrasonido que viajan diagonalmente a través de las paredes de la tubería. La diferencia de los tiempos es directamente proporcional al caudal (Figura 4). La precisión de este tipo de medidor alcanza el 0,5\% con intervalos de muestreo de por lo menos un segundo.

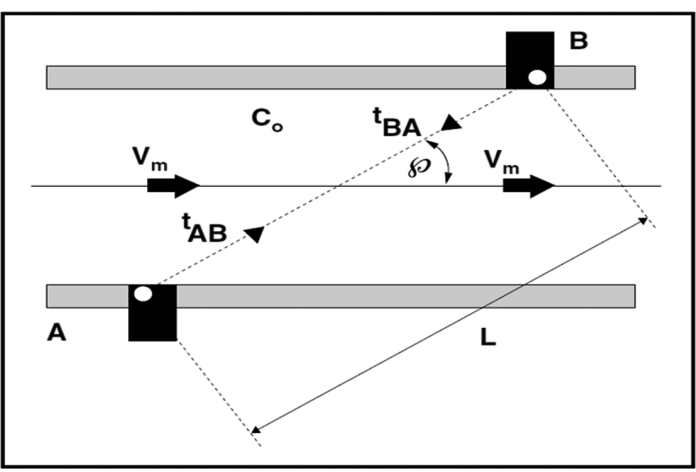

Figura 4. Ubicación de transductores en un medidor ultrasónico: A y B son los transductores; $\mathrm{V}_{\mathrm{m}}$ es la velocidad del flujo; $\mathrm{t}_{\mathrm{BA}} \mathrm{t}_{\mathrm{AB}}$ son los tiempos de tránsito de pulsos; $\mathrm{L}$ es la longitud diagonal; $\wp$ es el ángulo de la diagonal Fuente: Yoder [23]

La comparación entre los caudalímetros PD y los ultrasónicos se da en la tabla 4.

Tabla 4. Comparación entre caudalímetro de desplazamiento positivo y ultrasónico

\begin{tabular}{|c|c|c|c|}
\hline Categoría & Unidades & PD & Ultrasónico \\
\hline Tamaño & & $<1^{\prime \prime}-16^{\prime \prime}$ & $4 "-20 "$ \\
\hline \multirow{2}{*}{ Caudal máx. } & $\mathrm{BPH}$ & 12.500 & 50.000 \\
\hline & $\mathrm{m}^{3} / \mathrm{h}$ & 2.000 & 7.930 \\
\hline \multirow{2}{*}{$\begin{array}{l}\text { Presión } \\
\text { máx. }\end{array}$} & Psi & 1.440 & 1.440 \\
\hline & Bar & 100 & 100 \\
\hline Prover* & & $\begin{array}{l}\text { VTP, SVP, } \\
\text { CPP }\end{array}$ & $\mathrm{CPP}$ \\
\hline
\end{tabular}

*Volumetric Tank Prover (vTP), Small Volume Prover (svP) and Conventional Pipe Prover (CPP).

Fuente: elaboración propia

\subsubsection{Medidores de presión}

La medición de presión se realiza con el propósito de garantizar la seguridad del sistema y complementar la medición de caudal. La presión es esencial en la medición de gas natural debido a que es un fluido altamente compresible. La medición de presión se debe hacer localmente mediante manómetros y además con transmisores para llevar la señal al cuarto de control (ver figura 5). En la selección del instrumento, se deben considerar factores del proceso de medición como pulsación, vibración, purgas, dimensiones de las tuberías, gabinetes, soportes y tipo de conexión. Estas características varían si la medición es absoluta o diferencial.

Los filtros BS\&W requieren medir la presión diferencial para identificar el estado de impurezas recogidas, mientras que la medición de presión absoluta asociada al caudal se realiza aguas abajo del elemento primario (caudalímetro). La presión estándar para medición de volumen es de 14,69 PSI (101.325 KPa), según lo registrado por Rey [11] .

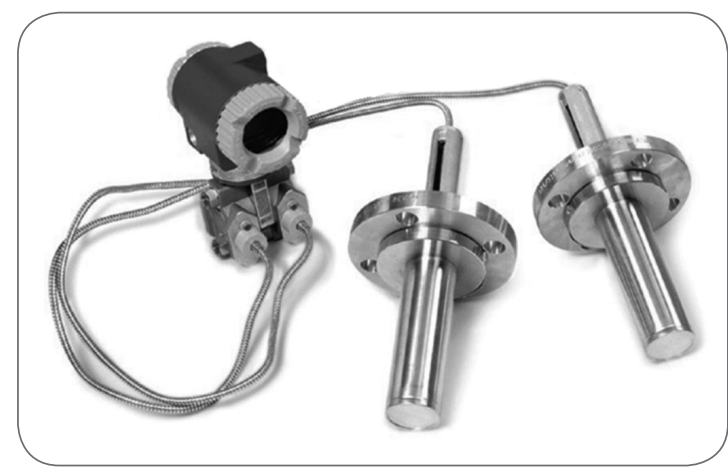

Figura 5. Sensor y transmisor de presión

Fuente: Krohne [31]

\subsubsection{Medidores de temperatura}

La medición de temperatura es muy importante para el proceso porque, al igual que la presión, afecta directamente el caudal volumétrico. Los instrumentos de medición de temperatura son generalmente termocuplas instaladas por medio de termopozos para protegerlas y permitir su rápido mantenimiento. A fin de obtener la medición de caudal, la temperatura del fluido se mide aguas abajo del caudalímetro. La temperatura estándar para medición de volumen es de $60,0^{\circ} \mathrm{F}\left(15,56^{\circ} \mathrm{C}\right)$ de acuerdo con las normas API y AGA [11]. 


\subsubsection{Computador de flujo}

Este equipo es un computador dedicado a la adquisición de datos de los instrumentos del sistema, y se compone de varios CPU de alta velocidad y capacidad de procesamiento. Todas las señales de instrumentación son dirigidas al computador de flujo para registrar y calcular el flujo volumétrico o másico. Los algoritmos y los cálculos de compensación para medir el flujo se basan en los estándares ISO, AGA y API. El computador de flujo puede funcionar de modo independiente o ligado a un sistema SCADA, generando reportes y almacenando las mediciones totales y parciales en bases de datos locales. Otra función importante es monitorear y controlar las válvulas de bloqueo y reguladoras de flujo, de acuerdo con Dupuis y Hwang [3].

\section{Distribución de la instrumentación}

La instrumentación para medición de caudal se distribuye de acuerdo con lo mostrado en la figura 6, que corresponde al diagrama de instrumentación y tuberías (P\&ID) para una estación de medición de gas de dos líneas. Hay una línea principal y otra idéntica que sirve de respaldo. El caudalímetro ultrasónico es el elemento primario, aguas abajo se miden la presión y la temperatura. Cada sensor tiene su respectivo transmisor. Los equipos para medición de calidad se observan en el diagrama a la entrada del fluido, aquí se encuentran el cromatógrafo, el analizador de humedad y el analizador de punto de rocío. Toda la instrumentación es comunicada con el computador de flujo, que a su vez es supervisado desde una HMI (interfaz hombre-máquina) y luego se comunica con el sistema SCADA.

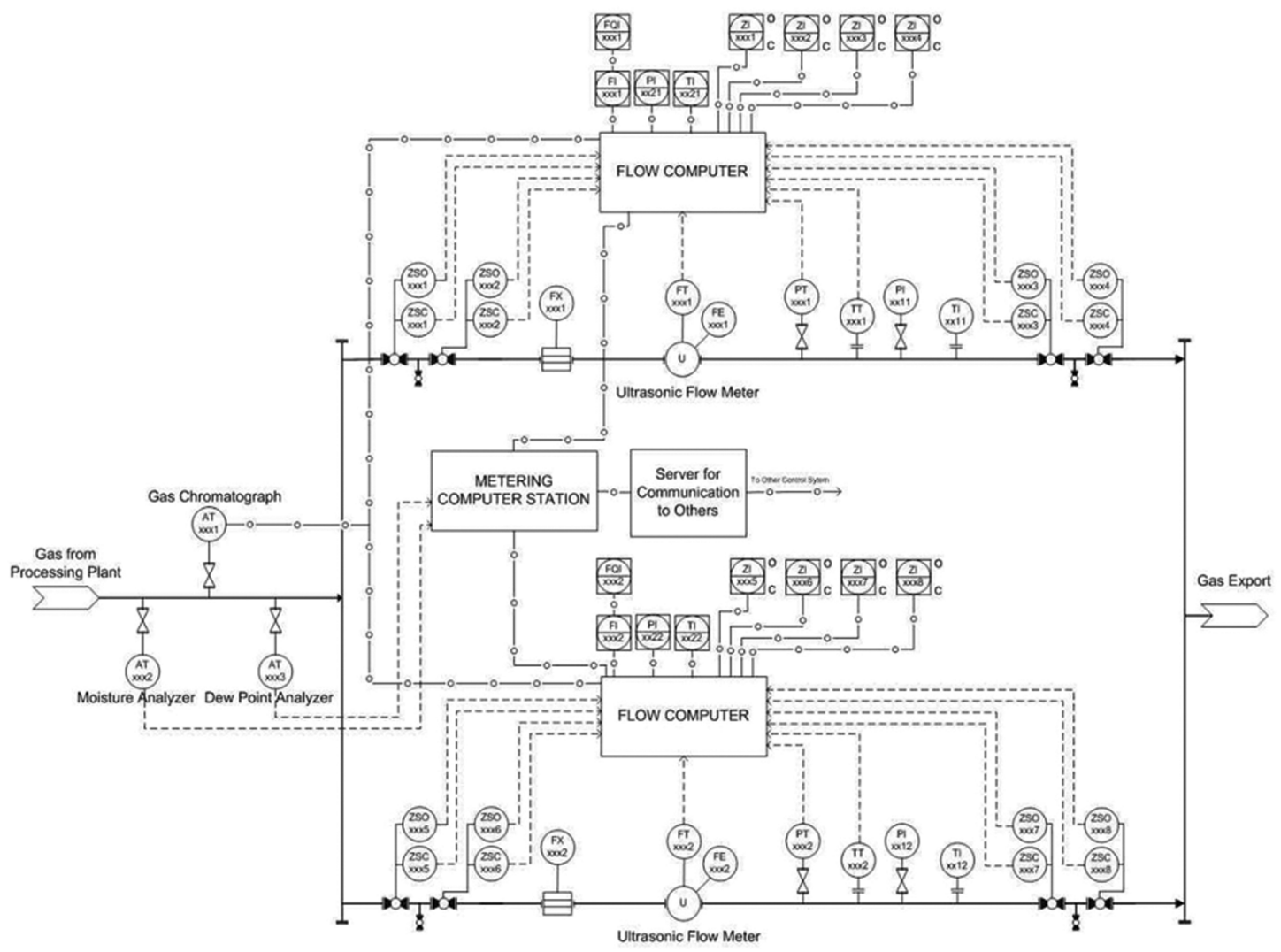

Figura 6. Distribución de instrumentos en un sistema de medición Fuente: Invensys Operations Management [32] 


\section{Conclusiones}

La TC en la industria de hidrocarburos es el procedimiento mediante el cual, con fines comerciales y/o fiscales, se determina la cantidad y la calidad de un hidrocarburo en un punto del sistema de transporte o sistema de distribución para que el vendedor ceda la exclusividad del control, el manejo y la posesión del fluido - quedando este bajo completa responsabilidad del comprador- y realice la respectiva facturación [33], [34]. Por tratarse de una medición que equivale a una cantidad de dinero, en la que el volumen del fluido dispensado está en el orden de los millones de pies cúbicos por día, el más pequeño error en la medición representa una pérdida económica significativa [35], por lo que se admiten errores bajos, menores al 0,1\% para hidrocarburos líquidos y menor al 0,5\% para gas natural.

Desde esta perspectiva, la medición estática de hidrocarburos se realiza con grandes y costosos tanques de almacenamiento, en donde la geometría del contenedor, la presión y la temperatura del fluido, y la homogeneidad del producto permiten determinar la cantidad almacenada. Los sistemas de medición dinámica no tienen almacenamiento, midiendo con equipos de instrumentación muy rápidos y precisos las características del fluido que varían fuertemente en el tiempo; el error total en la medición depende de una cadena de incertidumbres asociadas a cada instrumento. Los equipos de instrumentación deben ser de alta precisión, repetibilidad, resolución y velocidad de respuesta.

Debido a que las características de los combustibles cambian rápidamente y no son homogéneos durante la TC, los periodos de muestreo deben ser menores a 0,2 segundos. Para obtener estos resultados, se considera un computador de flujo como equipo central del sistema de medición; este registra la totalidad del caudal de la transferencia de custodia. Es un equipo basado en microprocesadores de alta capacidad que monitorea y controla en tiempo real las propiedades del hidrocarburo, ejecutando algoritmos especiales para determinar la calidad y la cantidad de flujo transferido. Los reportes del computador de flujo son utilizados para facturar el producto.

Por otro lado, la medición dinámica tiene ventajas sobre la medición estática, porque se facilita la manipulación por el menor tamaño. Se ahorra tiempo ya que no requiere que el fluido esté en reposo para hacer la medición y no hay error asociado a la geometría del contenedor. Además, para realizar calibración no se suspenden totalmente los instrumentos. Estos aspectos representan costos asociados a la disponibilidad y eficiencia del sistema. Una incorrecta medida podría suponer una evasión en el pago de impuestos o regalías [36], [37].

De acuerdo con lo expuesto, las unidades de medición son de tipo LACT (Lease Automatic Custody Transfer); para dicha medición, entonces, se usan equipamientos LACT de medición dinámica de alta precisión para realizar automáticamente la transferencia de custodia de hidrocarburos líquidos, con lo que se determina - de manera confiable- el volumen neto y la calidad del fluido. Estos sistemas se encuentran formados por un conjunto de equipos de instrumentación, válvulas y tuberías, montados y ensamblados rígidamente sobre una única estructura metálica denominada "metering skid" (patín de medición), apta para funcionar en la intemperie.

A su vez, el Instituto Americano del Petróleo (API), mediante los documentos API SPEC $11 \mathrm{~N}$ Specification for Lease Automatic Custody Transfer (LACT) Equipment [8] y el Capítulo 6 del Manual of Petroleum Measurement Standards: Metering Assemblies Section 1 Lease Automatic Custody Transfer (LACT) Systems [9], ha definido los requisitos óptimos para el diseño de estos sistemas a tasas por debajo de 11.000 barriles americanos veinticuatro horas al día, en aplicaciones de campo con presiones menores a los 500 psig (Pound per Square Inch Gauge $=\mathrm{lbf} / \mathrm{in}^{2}$ ).

Entre tanto, la unidad LACT obtiene el hidrocarburo de un tanque; el fluido es acondicionado eliminando el aire, el agua e impurezas presentes; el caudal, la presión, la temperatura y la densidad del fluido son medidos para calcular y registrar el volumen por medio del computador de flujo [38] Finalmente, se ajusta la presión de salida para dispensar el fluido. Esta metodología es actualmente adoptada para determinar sistemáticamente mediciones de alta exactitud, [39].

De acuerdo con lo anterior, las mediciones se deben realizar en condiciones estándar de presión y temperatura, según lo estipulan AGA y API. La normatividad al respecto es extensa y cubre los aspectos detallados para cada subproceso o equipo del sistema de medición; sin embargo, no existe una metodología definida para proponer el desarrollo de un sistema para transferencia de 
custodia que permita la evaluación para selección de instrumentación.

En consecuencia, la selección de instrumentación depende del tipo de medición requerida, y esto es definido por las características del proceso; en este sentido, los caudalímetros son los instrumentos más importantes del patín de medición y pueden obtener mediciones de volumen directo o de modo inferencial. Adicionalmente, requieren de mediciones complementarias de presión y temperatura para calcular el flujo.

Como perspectiva de trabajo, se pueden considerar otros caudalímetros de efecto - por ejemplo, el de Coriolis- o que determinan directamente el flujo másico de un fluido; así como los que utilizan algoritmos más sofisticados de medición - como las redes neuronales-. Para profundizar esta línea de investigación, puede estudiarse inicialmente, entre otra, la literatura dada en [40]-[50].

\section{Reconocimientos}

A los grupos de investigación SciBas y orcA, adscritos al Centro de Investigaciones y Desarrollo Científico de la Universidad Distrital Francisco José de Caldas (CIDC), por su asesoría en el modelamiento matemático y en la metodología documental.

\section{Referencias}

[1] British Petroleum, вP Statistical Review of World Energy. Londres: British Petroleum, 2012 [En línea]. Disponible en: https://www.laohamutuk.org/DVD/ docs/BPWER2012report.pdf.

[2] J. F. Arata, "Exportación de GNL. Liberando el potencial gasífero de Colombia", en XV Congreso Naturgas, Cartagena, Colombia, 2012.

[3] E. Dupuis y G. Hwang, "Custody Transfer: Flowmeter as Cash Register". Control Engineering Magazine, vol. 57, no. 9, p. 4, noviembre 2010.

[4] Comisión de Regulación de Energía y Gas (3 dic. 1999). Resolución 71 de 1999. Por la cual se establece el Reglamento Único de Transporte de Gas Natu-ral (RUT) [En línea]. Disponible en: http:// apolo. creg.gov.co/Publicac.nsf/Indice01/ Resoluci\%-C3\%B3n-1999-CREG071-99.

[5] O. Londoño, L. Maldonado y L. Calderón. Guía para construir estados del arte. Bogotá: International Corporation of Networks of Knowledge 2014.
[6] FMC Technologies Measurement Solutions, Inc., Measurement Products and Systems for the Oil and Gas Industry. Londres: FMC Technologies Measurement Solutions, Inc., 2012.

[7] American Petroleum Institute (API), Catalog: Publications, Programs and Services. Washington D.C. API, 2005 .

[8] American Petroleum Institute (API), SPEC 11N Specification for Lease Automatic Custody Transfer (LACT) Equipment. Washington D.C.: API, 1994.

[9] American Petroleum Institute (API), "Chapter $6 \mathrm{Me}-$ tering Assemblies - Section 1 Lease Automatic Custody Transfer (LACT) Systems", en MPMS - Manual of Petroleum Measurement Standards.

Washington

D.C.: API, 2002.

[10] M. Chaney, "Design, Operation, and Maintenance of LACT Units", en 85th International School of Hy-drocarbon Measurement, Oklahoma, Estados Uni-dos, 2010.

[11] American Petroleum Institute (API), ANSI/API Recommended Practice 551 - Process Measurement Ins-trumentation. Washington D.C.: API, 1993.

[12] C. V. Rey, "Seleccionar la tecnología adecuada para la medición de crudos pesados en transferencia de custodia", en Memorias III Congreso en Automatización de la Industria Petrolera JAIP, Bogotá, Colombia, 2012.

[13] Agencia Nacional de Hidrocarburos de Bolivia, "Glosario técnico" [En línea]. Disponible en: https:// www.anh.gob.bo/index.php?N=glosario.

[14] Ecopetrol S.A., "Calidad del gas natural en Colombia" [En línea]. Disponible en: https://www. ecopetrol.com.co/wps/portal/es/ecopetrol-web/ productos-y-servicios/productos/gas-natural/informacion-comercial/Calidad\%20del\%20Gas/Calidad + del + Gas

[15] S. Mokhatab, W. Poe y J. Speight. Handbook of Natu-ral Gas Transmission and Processing. Houston: Gulf Professional Publishing, 2006.

[16] G. McKeogh y K. Soleyn, "A Comparison of Moisture Measurement Technologies for Natural Gas", en 6th International Gas Analysis Symposium \& Exhibi-tion, Rotterdam, Netherlands, 2011.

[17] S. Hale. "The Fundamentals of Pipeline Gas Chromatographs". Pipeline \& Gas Journal, vol. 238, no. 7, 
[19] S. Mokhatab. "Fundamentals of Gas Pipeline Metering Stations". Pipeline \& Gas Journal, vol. 236, no. 1, 2009 [En línea]. Disponible en: https:// pgjonline.com/magazine/2009/january-2009vol-236-no-1/features/fundamentals-of-gas-pipeline-metering-stations.

[20] A. Creus, Instrumentación industrial. Barcelona: Alfaomega, 1997.

[21] D. J. Rudroff, "Design, Operation, and Maintenance of LACT Units", en 69th International School of Hydrocarbon Measurement, Houston, Texas, Estados Unidos, 1994 [En línea]. Disponible en: http://www. measurementlibrary.com/docs_library/events/ ishm1994/Docs/39.pdf.

[22] J. Yoder, "A Place for Positive Displacement PD Flowmeters Quietly Excel in Low-Flow Rate, High Viscosity, and Liquid and Gas Metering Applications", 2002 [En línea]. Disponible en: www.flowresearch. com/articles/PDF_Files/PD_Meters.pdf.

[23] J. Yoder, "Ultrasonic Flowmeters for Custody Transfer”, Flow Control Network, sept. 2010 [En línea]. Disponible en: https://www.flowcontrolnetwork. com/2010/09/26/ultrasonic-flowmeters-for-custody-transfer/.

[24] V. Hans y Y. Lin, "Self-Monitoring Ultrasonic Vortex and Correlation Gas Flow Meter", en 2005 IEEE Instrumentation and Measurement Technology Conference, Ottawa, Canadá, 2005.

[25] J. Reyes y A. Acevedo, "Simulation and Experimental Validation of a Transit Time in an Ultrasonic Gas Flow Meter Using Air", en 2010 IEEE ANDESCON, Bogotá, Colombia, 2010.

[26] D. W. Spitzer, "Selecting a Flowmeter for Custody-Transfer Applications", Flow Control Magazine, abr. 2011 [En línea]. Disponible en: https:// www.flowcontrolnetwork.com/2011/04/19/selecting-a-flowmeter-for-custody-transfer-applications/.

[27] K. Helminski, "Ultrasonic Meter Station Design Considerations", Daniel Measurement and Control White Paper, jul. 2010 [En línea]. Disponible en: http://www2.emersonprocess.com/ siteadmincenter/PM\%20Daniel\%20Documents/ WGMSC-2003-Gas-USM-Station-Design-Considerations-techWpaper.pdf.

[28] M. Kupnik y M. Gröschl, "Ultrasonic-based gas flowmeter for Harsh Environmental Conditions". $e$ \& $i$ Elektrotechnik und Informationstechnik, vol. 126 , no. 5, pp. 206-213, mayo 2009, doi: $10.1007 /$ s00502-009-0638-0.

[29] J. W. Bowen, "Multipath Ultrasonic Meters for Natural Gas Custody Transfer", Pipeline and Gas Journal, vol. 237, no. 4, 2010 [En línea]. Disponible en: https://pgjonline.com/magazine/2010/april-2010vol-237-no-4/features/multipath-ultrasonic-meters-for-natural-gas-custody-transfer.

[30] P. Lunde, K.-E. Froysa y M. Vestrheim, “Gerg Project on Ultrasonic Gas Flow Meters”, 2000 [En línea]. Disponible en: http://www.gerg.eu/public/uploads/ files/publications/technical_monographs/tm11.pdf

[31] Krohne, "Altosonic V12 - 12 Chord Ultrasonic Gas Flowmeters for Custody Transfer", s. f. [En línea]. Disponible en: https://krohne.com/en/products/ flow-measurement/flowmeters/ultrasonic-flowmeters/altosonic-v12/

[32] Invensys Operations Management, "Foxboro Pressure Transmitters" [En línea]. Disponible en: https:// www.schneider-electric.com/en/product-range/ 63509-pressure/3448261296-differential?subNodeId=3448270327en_WW

[33] Barchard Engineering Ltd., "Introduction to Gas Metering System, Instrumentation and Process Control", s. f. [En línea]. Disponible en: http://barchardengineering.com/_library/2016/10/introduction_to_gas_metering-1.pdf

[34] Comisión de Regulación de Energía y Gas (23 abr. 2008). Resolución 41 de 2008. Por la cual se modifica y complementa el Reglamento Único de Transporte de Gas Natural - RUT. [En línea]. Disponible en: http:// www.creg.gov.co/html/Ncompila/htdocs/Documentos/Energia/docs/resolucion_creg_0041_2008. htm.

[35] G. J. Covelli, "Facilidades tecnológicas para brindar trazabilidad a las mediciones de gases en Colombia”. Revista MET \& FLU, vol. 2, 2010 [En línea]. Disponible en: http://www.cdtdegas.com/images/ Descargas/Nuestra_revista/MetFlu2/metflu_No0206Mediciones_Gases.pdf.

[36] N. Quevedo, "Entre la bonanza petrolera y los organismos de control. Los dilemas de Pacific Rubiales", El Espectador, mar. 2012.

[37] V. Bynum, "Lаст Unit Proving - The Role of the Witness Class", en 85th International School of $\mathrm{Hy}$ drocarbon Measurement, Oklahoma, Estados Unidos, 2010 [En línea]. Disponible en: http://www. measurementlibrary.com/docs_library/events/ ishm2010/Docs/4080.pdf

[38] Endress+Hauser, "The Coriolis Flow Measuring Principle” [En línea]. Disponible en: https://www. id.endress.com/en/Field-instruments-overview/ Flow-measurement-product-overview/Coriolismass-flowmeters

[39] American Gas Association (AGA), AGA Report No. 11 Measurement of Natural Gas by Coriolis Meter. Washington D.C.: Transmission Measurement Committee, 2001. 
[40] M. Pereira, "Flow Meters: Part 1", IEEE Instrumentation \& Measurement Magazine, vol. 12, no. 1, pp. 18-26, febrero 2009, doi: 10.1109/ MIM.2009.4762948

[41] Y. Wang y Z. Li, “Temperature Compensation of Ultrasonic Flow Measurement Based on the Neural Network", en 2010 International Conference on Artificial Intelligence and Computational Intelligence, Sanya, China, 2010.
[42] I. Rauda y V. Ragasol, Sistemas electrónicos de medición de flujo para hidrocarburos en fase gaseosa. México D.F.: PEMEx, 2005 [En línea]. Disponible en: http://www.ri.pemex.com/files/ content/ACFE-MAxnaOYd.pdf

[43] R. C. Stubbs, General Arrangement Detail Layout. Houston: Southwestern Measurement Systems LLC, 2010.

[44] Y. Zee Ma, E. Gomez, Uses and abuses in applying neural networks for predictions in hydrocarbon resource evaluation, Journal of Petroleum Science and Engineering, Volume 133, 2015, Pages 66-75, ISSN 0920-4105, http://dx.doi.org/10.1016/ j.petrol.2015.05.006.

[45] A. Al-AbdulJabbar, S. Elkatatny, M. Mahmoud, \&amp; A. Abdulraheem, Predicting Rate of Penetration Using Artificial Intelligence Techniques. Society of Petroleum Engineers. (2018a, August 16). doi:10.2118/192343-MS.

[46] Y. Alcocer, \&amp; P. Rodrigues, Neural Networks Models for Estimation of Fluid Properties. Society of Petroleum Engineers. (2001, January 1). doi:10.2118/69624-MS.

[47] A. Clifford, \&amp; F. Aminzadeh, Gas Detection From Absorption Attributes And Amplitude Versus Offset With Artificial Neural Networks In Grand Bay Field. (2011, January 1). Society of Exploration Geophysicists.

[48] P.S. Hegeman, C., Dong, C., N. Varotsis, \&amp; V. Gaganis, . Application of Artificial Neural Networks to Downhole Fluid Analysis. Society of Petroleum Engineers. (2009, February 1). doi:10.2118/123423-PA.

[49] E. Salaheldin, T. Zeeshan, M. Mahmoud, Real time prediction of drilling fluid rheological properties using Artificial Neural Networks visible mathematical model (white box), Journal of Petroleum Science and Engineering, Volume 146, 2016, Pages 1202-1210, ISSN 0920-4105, http:// dx.doi.org/10.1016/j.petrol.2016.08.021.

[50] J. Garrido, P. Reina, M. Vázquez, A. García, Control de Nivel a través de Redes Neuronales. Universidad Tecnológica del Sureste de Veracruz, 2009. 